\title{
Severe Wolfram Syndrome Caused by a Novel Frameshift Mutation in WFS1 Gene: Effect on the WFS1/CaM Interaction and Phenotype-Genotype Correlation
}

\author{
Mouna Tabebi1,2, Rahma Felhi' ${ }^{1}$, Houcem Elomma Mrabet ${ }^{3}$, Wajdi Safi ${ }^{4}$, Baha Zantour ${ }^{3}$, \\ Mohamed Habib Sfar ${ }^{3}$, Mohammed Abid4, Mouna Mnif Feki", Faiza Fakhfakh ${ }^{{ }^{*}}$ \\ ${ }^{1}$ Laboratory of Molecular and Functional Genetics, Faculty of Science, University of Sfax, Tunisia \\ ${ }^{2}$ Laboratory of Human Molecular Genetics, Faculty of Medicine, University of Sfax, Tunisia \\ ${ }^{3}$ Department of Endocrinology, Tahar Sfar Hospital, Mahdia, Tunisia \\ ${ }^{4}$ Department of Endocrinology, Hedi Chaker Hospital, Sfax, Tunisia
}

How to cite this paper: Tabebi, M., Felhi, R., Elouma Mrabet, H., Safi, W., Zantour, B., Sfar, M.H., Abid, M., Mnif-Feki, M. and Fakhfakh, F. (2021) Severe Wolfram Syndrome Caused by a Novel Frameshift Mutation in WFS1 Gene: Effect on the WFS1/CaM Interaction and Phenotype-Genotype Correlation. Open Journal of Genetics, 11, 77-92. https://doi.org/10.4236/ojgen.2021.114008

Received: October 13, 2021

Accepted: November 20, 2021

Published: November 23, 2021

Copyright $\odot 2021$ by author(s) and Scientific Research Publishing Inc. This work is licensed under the Creative Commons Attribution International License (CC BY 4.0).

http://creativecommons.org/licenses/by/4.0/

\begin{abstract}
Mutations in the WFS1 gene have been reported in Wolfram syndrome (WFS), a rare and autosomal recessive disorder defined by early onset of diabetes mellitus and progressive optic and hearing impairment. Only few data are available concerning the association between clinical and molecular aspects of the WFS. We present a consanguineous family with a patient presenting an early onset of WFS and severe manifestations. Sequencing of WFS1 gene was performed for all the family members to search for responsible mutation and bioinformatics tools were conducted to predict its effect on structure and function of the protein. We have detected a novel frameshift mutation in the proband at homozygous state and at the heterozygous state in the parents who have no WFS manifestations. In silico analysis predicted the pathogenicity of the mutation and could lead to a complete loss of its function. Thus, 3D modeling showed that the mutation abolishes the interaction of the CaM binding region to the N-terminal of WFS1 and then impairs the WFS1-CaM complex formation. Genotype-phenotype correlation study shows that the novel mutation predisposes to early onset of diabetes and severe symptoms observed in the proband. We also report the effect of the frameshift mutation on the CaM-WFS1 impaired binding, and we discuss its possible consequence in pancreatic $\beta$-cells dysfunction and its role in the early onset of diabetes. In conclusion, the combination of impaired functions of WFS1 including unproper interaction of the $\mathrm{CaM}, \mathrm{Ca}^{2+}$ uptake, mitochondrial dysfunction, and apoptosis under the ER stress could be involved in the
\end{abstract}


severe phenotype and early onset of WFS of our patient.

\section{Keywords}

Wolfram Syndrome, WFS1, Frameshift Mutation, WFS1-CaM Binding

\section{Introduction}

Wolfram syndrome (WFS) (MIM 222300) is a rare hereditary disorder described for the first time in 1938, with an estimated prevalence worldwide from $1 / 55.000$ to $1 / 770.000$ [1]. It is a progressive neurodegenerative disease, started with diabetes mellitus (DM) and optic atrophy (OA) in the first decade followed by diabetes insipidus and hearing impairment (HI) in the second decade, by neuropathic bladder in the third decade, and could be followed by neurological complications in the fourth decade [2]. Nonetheless, other neurological and psychiatric manifestations could be observed such as ataxia and peripheral neuropathy [3]. The inheritance mode of WFS is usually autosomal recessive caused mainly by mutations in WFS1 gene mapped to chromosome 4p16.1 [4]. WFS1 gene contains eight exons encoding a transmembrane 890 -amino acid protein called wolframin an N-glycosylated ER (endoplasmic reticulum) membrane protein [5]. The Wolframin protein (WFS1) is composed of an $\mathrm{N}$-terminal cytoplasmic domain, nine transmembrane segments, and C-terminal luminal domain [6]. It is has been likely involved in several functions including its role in the unfolded protein response within the ER lumen, which is essential to cellular homeostasis and integrity [7]. On the other hand, Wolframin has been identified to be one of the calmodulin (CaM) targets and interacting in a $\mathrm{Ca}^{2+}$ dependent manner [8] [9].

To date, little is known about Wolfram syndrome and few studies in small patient cohorts, have described mutations in the WFS1 gene, distributed mainly on C-terminal domain [10]. Previous studies provided some evidence for a genotype-phenotype correlation [11] [12] and reported that WFS1 loss of function caused by frameshift and nonsense mutations, lead to glucose intolerance and progressive pancreatic $\beta$ cells apoptosis, via ER stress activation pathway [13] [14] [15] [16].

We present a family including a patient with an early onset of WFS and severe manifestations. The result of WFS1 gene sequencing showed a novel homozygous frameshift mutation leading to loss of function. Bioinformatic tools revealed that this mutation leads to a truncated protein and molecular modeling showed that this mutation disturbs the WFS1-CaM interaction. Moreover, we review additional previously described truncated mutations leading to complete or partial loss of function of WFS1 and we compared the resulting patient's phenotype.

\section{Material and Methods}

\subsection{Subjects}

We studied a consanguineous family where the daughter presents severe wol- 
fram syndrome. The minimum diagnostic criteria for WFS were childhood occurrence of diabetes mellitus (DM) and optic atrophy (OA).

Written consent to participate in the study was obtained from the involved individuals in this study. The probands underwent comprehensive physical exams, including endocrinological, ophthalmological, and neurological assessments.

In addition, 100 healthy individuals from the same ethnocultural group were tested as controls. These controls should have neither personal nor family history of diabetes or any other disorder. All controls provided informed consent.

\subsection{Methods}

\subsubsection{DNA Extraction}

Total DNA was extracted from peripheral blood leucocytes using phenolchloroform standard procedures [17].

\subsubsection{WFS1 Gene Sequencing}

For the analysis of WFS1 gene (NM_006005), all exons and their encompassing intronic regions were amplified using specific primers (Table 1), by PCR reaction in a thermal cycler (GenAmp PCR System 9700; Applied Biosystem). PCR reactions were performed in $25 \mu \mathrm{l}$ reaction mixtures containing $100 \mathrm{ng}$ of total DNA, $10 \mu \mathrm{M}$ of each primer, $2 \mathrm{mM} \mathrm{MgCl} 2,10 \mathrm{mM}$ dNTP, $1 \times$ PCR buffer and 2 $\mathrm{U}$ Taq DNA polymerase. The reaction mixture was incubated at $94^{\circ} \mathrm{C}$ for $5 \mathrm{~min}$

Table 1. Primers sequences used for PCR-Sanger sequencing.

\begin{tabular}{|c|c|c|c|}
\hline Exons & Sequence & PCR product & Annealing temperature \\
\hline 2 & $\begin{array}{l}\text { F: 5' GATCCTGTATGGAGTGTCTGGC 3' } \\
\text { R: 5'GCTGAACTGCAGAGGACCTGGC3’' }\end{array}$ & $473 \mathrm{pb}$ & $65^{\circ} \mathrm{C}$ \\
\hline 3 & $\begin{array}{l}\text { F: 5' AGGCAAACAGTGGCTTTCTGGG 3' } \\
\text { R: 5' ATGGGGCTCAAGTGGGGTAGGG 3' }\end{array}$ & $415 \mathrm{pb}$ & $65^{\circ} \mathrm{C}$ \\
\hline 4 & $\begin{array}{l}\text { F: 5' CTGGTGTGACCCCATTTCTGCC 3' } \\
\text { R: } 5^{\prime} \text { CTTGGAGATACCAACACAAGCC } 3\end{array}$ & $518 \mathrm{pb}$ & $65^{\circ} \mathrm{C}$ \\
\hline 5 & $\begin{array}{l}\text { F: 5' CAGATGTCCATGCATCCTTCCC 3' } \\
\text { R: 5’ CTGTCCTCACAGGGAAGGTCCC3’' }\end{array}$ & $462 \mathrm{pb}$ & $65^{\circ} \mathrm{C}$ \\
\hline 6 & $\begin{array}{l}\text { F: 5' ATCCCCAGAACGTAGGATGCCC } 3^{\prime} \\
\text { R: } 5^{\prime} \text { CAGCGTCCAGAACACTGAGCCC } 3\end{array}$ & $421 \mathrm{pb}$ & $65^{\circ} \mathrm{C}$ \\
\hline 7 & $\begin{array}{l}\text { F: 5' AGCTCCTTTCTTAGCTTGGCCC 3' } \\
\text { R: 5' CTTTGTTAGGCGCGAACCTCCC 3' }\end{array}$ & $372 \mathrm{pb}$ & $65^{\circ} \mathrm{C}$ \\
\hline $8-1$ & $\begin{array}{l}\text { F: 5' TGGTGATGGGAAAACGCAAGGG 3' } \\
\text { R: } 5^{\prime} \text { ACATGAAGCACACCAGGTAGGG 3' }\end{array}$ & $884 \mathrm{pb}$ & $65^{\circ} \mathrm{C}$ \\
\hline $8-2$ & $\begin{array}{l}\text { F: 5' CCCATGCAGAGCCCTACACGCG 3' } \\
\text { R: 5' GCTTGGCCAGCAGCTTAAGGCG 3' }\end{array}$ & $942 \mathrm{pb}$ & $66^{\circ} \mathrm{C}$ \\
\hline $8-3$ & $\begin{array}{l}\text { F: 5' CCTGGAAGGAGACCAACATGGC 3' } \\
\text { R: } 5 \text { ' TCAGGCTCAGGGTGGAGATGGC 3' }\end{array}$ & $887 \mathrm{pb}$ & $68^{\circ} \mathrm{C}$ \\
\hline $8-4$ & $\begin{array}{l}\text { F: 5' TTTTCTTCCCATTCCTGTCGGC 3' } \\
\text { R: 5’ TTCATTCAACAGGAGTGCAGGC 3' }\end{array}$ & $911 \mathrm{pb}$ & $65^{\circ} \mathrm{C}$ \\
\hline
\end{tabular}


for denaturation, followed by 30 cycles of $94^{\circ} \mathrm{C}$ for $40 \mathrm{~s}$, annealing temperature for $40 \mathrm{~s}$ (Table 1), $72^{\circ} \mathrm{C}$ for $40 \mathrm{~s}$, and a final extension of $10 \mathrm{~min}$ at $72^{\circ} \mathrm{C}$. The PCR products were separated by electrophoresis on a $2 \%$ agarose gel stained with ethidium bromide. The PCR products were purified and directly Sanger sequenced on both strands using ABI PRISM 1.1 Big Dye terminator chemistry (Applied Biosystems) on the ABI 3100 Genetic Analyzer (Applied Biosystems). The blast homology search was performed using the program available at the National Center for Biotechnology Information website in comparison with the updated consensus Cambridge sequence (GenBank Accession No. NC_000004.12). Sanger sequencing was used also to perform the segregation analysis within the family.

\subsubsection{Sequence Alignment and Pathogenicity Prediction}

The sequence alignment of the WFS1 gene was performed using the ClustalW program (https://www.ebi.ac.uk/Tools/msa/clustalo/).

To investigate the pathogenicity of the novel WFS1 variant, VarSome software was used https://varsome.com, V6.7, Lausanne, Switzerland). Varsome is a powerful annotation tool and search engine for human genomic variants [18]. For novel variant, Varsome provides multiple evidence supporting the benignity or pathogenicity and the verdict is reported according to the American College of Medical Genetics (ACMG) guidelines.

The assessment of the possible impact of an amino acid substitution on the protein structure and the possible effect on the protein function was performed using Protter (https://wlab.ethz.ch/protter/start/), Prot-param program (https://web.expasy.org/protparam/). Finally, MetaDome (https://stuart.radboudumc.nl/metadome/) used the gnomAD for population variations and ClinVar for pathogenic variants and allowed to create genetic intolerance regions at the amino acid resolution for whole human protein domains.

\subsubsection{In Silico Protein Analysis}

We focused ours in silico investigations on the p.A214SfsXr72 variant. There are currently no 3-D crystal structures for any part of the WFS1 protein. Even in SWISS-MODEL server, no identified templates were found for comparative modeling, since the sequence of the ER lumenal domain containing the wanted residues was not submitted. Therefore, RaptorX Structure Prediction program has been an alternative that servers for predicting protein secondary and tertiary structure, binding site, and GO annotation (http://raptorx.uchicago.edu/). The superposition of the wild type and mutated models ensures the calculation of the RMSD and the PyMOL software (V2.4.1) performed molecules interactions.

The 3-D crystal structure model of the human CaM was available (PDB code 2jc6). Wolframin residues (2-285) are the interaction region with CaM. The docking study starts with the definition of a binding site. The size and location of this binding site are adjusted interactively and visualized in PyMOL. Subse- 
quently, the necessary files for the receptor definition and ligand are generated. The docking calculations and the results are visualized from within PyMOL. Docking results shows the 3-D structure and location of CaM binding to the WFS1 protein and their interactions.

\section{Results}

\subsection{Clinical Findings}

The proband is a 30 -year-old female, with a previous history of diabetes mellitus treated with insulin since the age of 3 years presented to her usual follow-up at the endocrinology outpatient clinic. She consulted at the age of 7 years to the ophthalmology department because of progressive loss of vision, and she was diagnosed with optic atrophy. At the age of 20 years old, she reported a progressive bilateral loss of hearing, and audiometric examination showed a bilateral high-frequency hearing impairment. The patient also reported dysuria and intermittent urinary incontinence since the age of 18 years old, which were related to a neurogenic bladder and pyelocaliceal dilation (PCD). The patient reported persistent polydipsia even though her diabetes was controlled, and further exploration was in favor of a central diabetes insipidus. Neurological examination showed cerebellar ataxia with autonomic neuropathy or dysautonomia affecting the nerves VIII, IX, and X. The patient also reported difficulties of swallowing, for which a digestive endoscopy and manometry were conducted and showed gastroduodenal hypokinesia. There were no similar cases in her family, and her parents and her sister had normal blood glucose levels, and fundus examination showed no optic atrophy (Figure 1(a)).

\subsection{Genetic Study and Pathogenicity Prediction}

The molecular analysis of the WFS1 gene in the patient revealed a novel homozygous frameshift mutation c.639_642delGGCG in exon 6 (p.A214SfsX72). The mutation was inherited from her heterozygous parents, who were first cousins, and was absent in her sister (Figure 1(b)) and 100 sequenced controls. The Multiple sequence alignment across several species revealed that the p.A214SfsX72 mutation is located in an evolutionarily conserved position of WFS1 protein (Figure 1(c)).

The novel frameshift c.639_642delGGCG in exon 6 of WFS1 gene leads to a premature stop codon (p.A214SfsX72) and according to the Varsome program it causes the total removal of the nine transmembrane domains in a short nonfunctional protein of 286 amino acids instead of 890 amino acids (Figure 1(d)). The remaining 286 aa of the N-terminal represents a truncated WFS1 protein, which is likely to be degraded after translation. MetaDome software showed that this residue is an intolerance region for any mutation. Besides, the ProtParam program showed a 1.7 -fold increase of the instability index in the mutated protein. These bioinformatics tools predicted that p.639_642delGGCG is most likely damaging the WFS1 protein. 
(a)

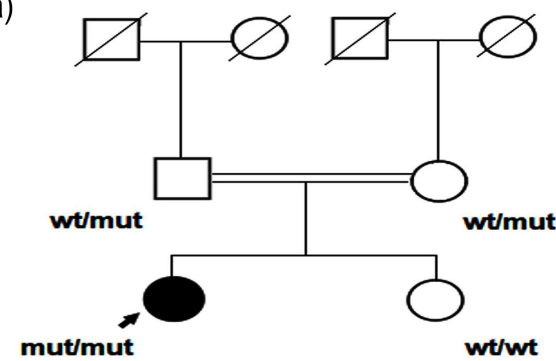

(b)

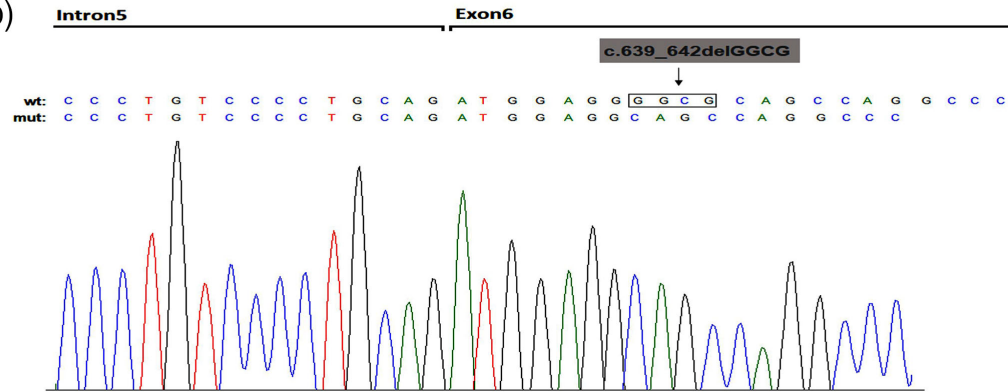

(c)

Human sreradgtgptkgdmeipfeevlerakagdpkaqtevgkhylqlagdtde elnsctavdwlvlaakggrreavkllrrcladrrgitsenerevrqlssetdleravrkaalvmy Rat sregtdkagomkadveipfeeilekakagdpkaqtevgkhylrlandadeelnscsavawlilaakggrreavkllrrcladrkgitseneaevkalssetdleravrkaalvmy $\psi k$ lnpkkkkgvavsellenvggvneqdggaapgpvpks lqkgrrmlerlvs Mouse sreetdragpmkadveipfeevlekakagdpkaqtevgkhylrlandadeelnscsavawlilaakggrreavkllrrcladrkgitseneaevkqlssetdleravrkaalvmy 4 lnpkkkkqvavsellenvgquneqdggaapgpvpks lqkgrrmlerlvs Bovine srermdgtgpakgdvdtpfeevlekakagdpkaqtevgkhylqlagdgdeelnnctavdwltlaakqgrreavkllrrcladrrgitsenegevrqlssetdleravrkaalvmy $4 k$ lnpkkkkqvavaellenvgqunehdggvapgpvpkslgkgrrvlerlvs

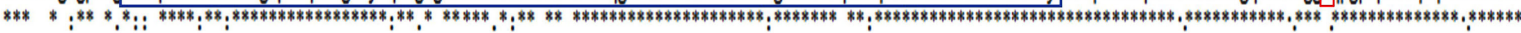

(d)

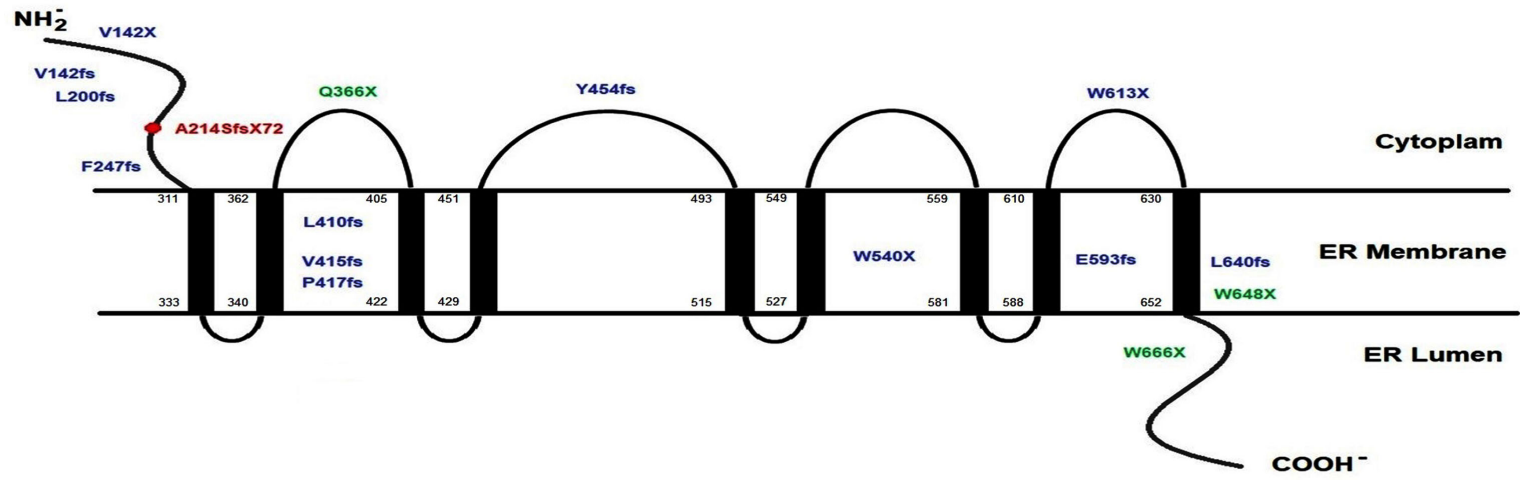

Figure 1. (a) Pedigree of WFS family. Black circle: affected female; white square: unaffected male; white circle: unaffected female; arrow: the proband. (b) Sanger sequencing results of WFS1 gene. The proband has a novel frameshift mutation at codon 214 (p.A214SfsX72) in exon 6 of WFS1 caused by the homozygous deletion mutation (c.639_642delGGCG). (c) Multiple sequence alignment of N-terminal region of WFS1 from different species (Human, Rat, Mouse Bovine) using ClustalW. The red background indicated mutated residue. The blue background indicated CaM-binding region of wolframin protein (90-186). (d) The position of the nonsense and frameshift mutations in WFS1. Predicted structure of the wolframin with nine putative transmembrane domains, and position of the novel mutation in WFS1 indicated by the red dot. Mutations are color-coded according to their mutation groups: complete loss of function (blue) and partial loss of function (green).

\subsection{Effect of the p.A214SfsX72 Mutation on the 3D WFS Protein Structure and Its Interaction with CaM Protein}

The 3-D modeling of the wild type and mutated WFS1 proteins showed the absence of the first helix (residues 67 - 77) in the mutated protein. Also, the root means square deviation or RMSD was significantly high $(7.58 \AA>2)$ suggesting the overall modified structure of the mutated WFS1 protein compared to the wild type (Figure 2).

The proteomic analysis identified that wolframin is one of the Calmodulin (CaM) binding protein targets [8]. Therefore, the N-terminal cytoplasmic domain of WFS1 protein (residues 2-285) contains its interaction region with CaM protein indicating that the p.A214SfsX72 mutation could affect the CaM binding ability of WFS1 protein. Indeed, we suggest that p.A214SfsX72 mutation abolishes this binding since the missing helix present multiple interactions within the neighborhood amino acids and the CaM protein (Figure 2). 


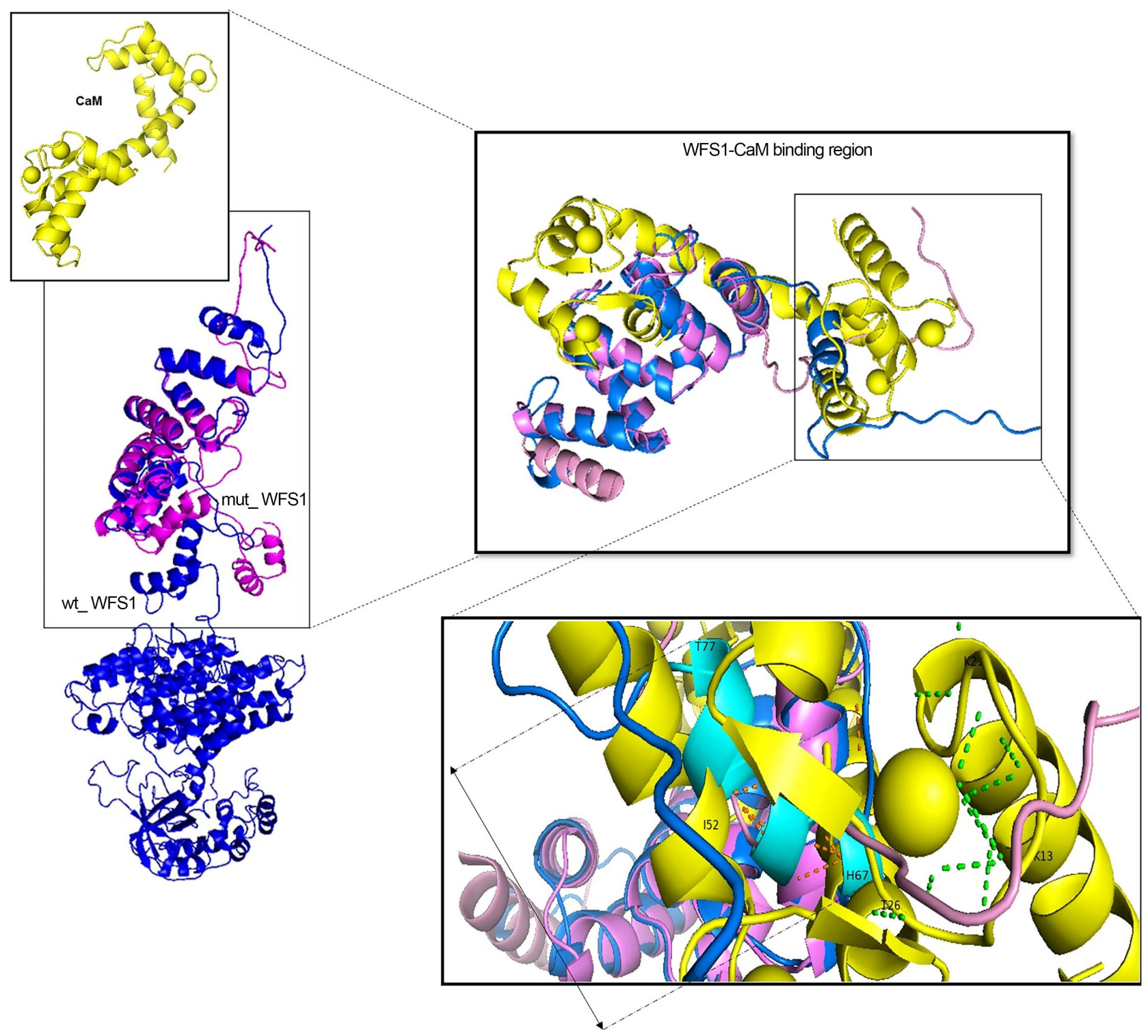

Figure 2. Cartoon representation of the model structure of wild-type (pink color) and mutated (blue color) WFS1 and CaM (yellow color). Left: Overall structure and superposition of the two WFS1 models. Right: N-terminal WFS1 region and CaM binding region. Zoom on the interaction between wt-WFS1 and CaM binding region (orange dotted line) and mutated-WFS1 and Ca-M binding region (green dotted line). Missed helix (residues 67 - 77) in the mutated model colored with blue color.

\subsection{Genotype-Phenotype Correlation Analysis}

We generated a mutational spectrum of nonsense and frameshift mutations of the WFS1 according to Human Gene Mutation Database (HGMD) (http://www.hgmd.cf.ac.uk/ac/index.php). We selected 15 WFS1 known mutations, including 6 nonsense and 9 frameshift mutations in previously described patients with Wolfram syndrome, mainly located in exon 8 (Table 2). Exon 8 is the longest WFS1 exon with $1.812 \mathrm{Kbp}$ and encodes the nine transmembrane domains and the C-terminal extracellular region of wolframin (Figure 1(d) and Table 2). We aimed to study the genotype-phenotype correlation. For this purpose, we divided the 15 mutations according to the predicted consequences on 
Table 2. Nonsense and frameshift mutations with complete and partial loss of WFS function.

\begin{tabular}{|c|c|c|c|c|c|c|c|}
\hline Mutation group & $\begin{array}{l}\text { Age of } \\
\text { onset }\end{array}$ & Exon & $\begin{array}{l}\text { Nucleotide } \\
\text { change }\end{array}$ & $\begin{array}{l}\text { Amino acid } \\
\text { change }\end{array}$ & $\begin{array}{l}\text { Type of } \\
\text { mutation }\end{array}$ & $\begin{array}{l}\text { Homo/ } \\
\text { Hetero }\end{array}$ & $\begin{array}{l}\text { First } \\
\text { description }\end{array}$ \\
\hline Complete loss of function & 1.8 & $4+8$ & $\begin{array}{l}\text { c.409_424dup + } \\
\text { c.1193_1243dup }\end{array}$ & $\begin{array}{l}\text { p.V142X + } \\
\text { p.V415fs }\end{array}$ & $\begin{array}{l}\text { Nonsense }+ \\
\text { Frameshift }\end{array}$ & Homo & {$[12]$} \\
\hline Complete loss of function & 4.3 & 5 & c.599delT & p.L200fs & Frameshift & Homo & [12] \\
\hline Complete loss of function & 1.5 & 7 & c.740_741delTT & p.F247fs & Frameshift & Homo & {$[12]$} \\
\hline Complete loss of function & 1.7 & 8 & c. $1096 \mathrm{C}>\mathrm{T}$ & p.Q366X & Nonsense & Homo & {$[1]$} \\
\hline Complete loss of function & 1 & 8 & c.1362_1377del16 & p.Y454fs & Frameshift & Homo & {$[31]$} \\
\hline Complete loss of function & 1.3 & 8 & c. $1619 \mathrm{G}>\mathrm{A}$ & p.W540X & Nonsense & Homo & {$[12]$} \\
\hline Complete loss of function & 4.2 & 8 & p.1775_1776insGGAT & p.E593fs & Frameshift & Homo & {$[12]$} \\
\hline Complete loss of function & 5.1 & 8 & c. $1838 \mathrm{G}>\mathrm{A}$ & p.W613X & Nonsense & Homo & {$[2]$} \\
\hline Complete loss of function & 3.3 & 8 & c.1919_1929del & p.L640fs & Frameshift & Homo & {$[12]$} \\
\hline Complete loss of function & 3 & 6 & c.639_642del & p.A214Sfs & Frameshift & Homo & Our study \\
\hline Partial loss of function & 2.6 & $7+8$ & $\begin{array}{l}\text { c. } 740 \_741 \text { delTT }+ \\
\text { c. } 2048 \mathrm{~T}>\mathrm{C}\end{array}$ & $\begin{array}{l}\text { p.F247fs + } \\
\text { p.M683R }\end{array}$ & $\begin{array}{l}\text { Frameshift + } \\
\text { missense }\end{array}$ & \multicolumn{2}{|c|}{ Comp Hetero [12] } \\
\hline Partial loss of function & 3.7 & $5+8$ & c.599delT + c.2006A > G & $\begin{array}{l}\text { p.L200fs + } \\
\text { Y669C }\end{array}$ & $\begin{array}{l}\text { Frameshift }+ \\
\text { missense }\end{array}$ & \multicolumn{2}{|c|}{ Comp Hetero [1] } \\
\hline Partial loss of function & 4 & 8 & $\begin{array}{l}\text { c. } 1251 \_1252 \text { delinsG }+ \\
\text { c.1973delA }\end{array}$ & $\begin{array}{l}\text { p.F417fs + } \\
\text { p.K658fs }\end{array}$ & Frameshift & \multicolumn{2}{|c|}{ Comp Hetero [12] } \\
\hline Partial loss of function & 8.5 & 8 & c. $1943 \mathrm{G}>\mathrm{A}$ & p.W648X & Nonsense & Homo & {$[32]$} \\
\hline Partial loss of function & 4.9 & 8 & c. $1997 \mathrm{G}>\mathrm{A}$ & p.W666X & Nonsense & Hetero & [33] \\
\hline
\end{tabular}

Homo: Homozygous; Hetero: Heterozygous; Comp Hetero: Compound Heterozygous; (?): Unknown.

wolframin function [5] into a group with partial loss of function and a group with complete loss of function in which we included our homozygous frameshift mutation (p.A214SfsX72).

Comparison of age at onset of WFS between these two groups revealed that mean age at WFS onset was $2.71 \pm 1.52$ years in individuals carrying mutations with a predicted complete loss of function, $4.74 \pm 2.25$ years in those carrying mutations with a predicted partial loss of function.

Using the following approach, we showed that the mutation's nature and its consequence on WFS1 function could define the WFS age at onset. Unfortunately, clinical data were incomplete and did not allow any statistically significant correlation between the two different groups of mutations and other clinical features of the WFS.

\section{Discussion}

\section{1. p.A214SfsX72 Responsible for WFS1 Loss of Function}

Wolfram syndrome is an autosomal recessive rare hereditary neurodegenerative disease. The Juvenile-onset of DM and OA is the current ascertainment criteria for WFS [19]. WFS1 gene was discovered in 1998 and described as responsible for most WFS [10]. Till now, more than 300 different mutations spread over the 
entire coding region in WFS1 have been identified, but most of these mutations are located in the exon 8 encoding the nine transmembrane domains and the C-terminal tail of wolframin [20]. Mutations in WFS1 gene are typically inactivating, suggesting that a loss of function causes the disease phenotype [21].

In this study, we identify a novel mutation located in exon 6 of WFS1 c.639_642delGGCG, (p.A214SfsX72) which was disease-causing only in its homozygous status in the patient and his heterozygous parents did not present any feature of WFS. The mutation p.A214SfsX72 was within an evolutionarily conserved region in $\mathrm{N}$-terminal tail and bioinformatics tools predicted it to be deleterious. Indeed, it causes a frameshift and leads to a premature stop codon at position 286 producing a truncating protein in which all the domains were removed and retains only the $\mathrm{N}$ terminal tail. This mutation leads to loss of function based on computational analysis and compared with previously reported frameshift and nonsense mutations (Table 2).

\subsection{Severe Phenotype Correlated to p.A214SfsX72}

Related to complete or partial loss of function, patients could present phenotype with variable severity. Our proband with severe phenotype presented diabetes mellitus early at the age of 3 years followed by bilateral optic atrophy at the age of 7 years and hearing impairment at the age of 20 years. Therefore, our patient was diagnosed with DM much earlier than OA and hearing loss. Compared with previously reported patients with frameshift, nonsense and missense mutations and for whom clinical features details were reported (Table 3 ) our proband presented the earliest diabetes age of onset. The genotype-phenotype analysis showed that age at onset of WFS among the individuals carrying mutations with complete loss of function is earlier than those carrying mutations with partial loss of function that agrees with our in-silico prediction for the proband having an early age of onset. This finding lines the meta-analysis data by Cano et al. [11], suggesting that mutations leading to a loss of function predispose to an earlier onset of diabetes. On the other hand, additional symptoms were also reported in most severe WFS phenotypes including, cataract and neurological defect [10] [22], but our patient seems presented more complicated and severe phenotype with cerebellar ataxia and renal and gastric dysfunctions which could be related to the type and effect of the responsible p.A214SfsX72 mutation. Thus, and based on clinical data availability related to the details of phenotype and the loss of function of WFS1 protein, we picked five WFS1 mutations including two frameshifts (F883fsX951 and F883fsX950) and two nonsense (p.Q486X and Q194X) mutations (Table 3). Our data analysis revealed that the two selected frameshift mutations led to mutated proteins carrying an extended C-terminal region and that p.Q486X mutation led to a short protein with only 3 out of 9 transmembrane domains. However and as the Q194X mutation, our novel frameshift mutation p.A214SfsX72 generates only a part of the N-terminal of WFS1 protein and which is probably responsible for the very severe phenotype of our proband (Figure 3). 
Table 3. Clinical features associated to the WFS1 mutated gene in our study compared to the litterature.

\begin{tabular}{|c|c|c|c|c|c|c|c|c|c|}
\hline Case & $\begin{array}{l}\text { WFS1 } \\
\text { loss of } \\
\text { function }\end{array}$ & $\begin{array}{l}\text { Age } \\
\text { (years) }\end{array}$ & Sex & $\begin{array}{l}\text { Family } \\
\text { history }\end{array}$ & $\begin{array}{l}\text { DM, age of } \\
\text { diagnosis }\end{array}$ & $\begin{array}{l}\text { OA, age of } \\
\text { diagnosis }\end{array}$ & $\begin{array}{l}\text { HI, age of } \\
\text { diagnosis }\end{array}$ & $\begin{array}{l}\text { Other features, } \\
\text { age of diagnosis }\end{array}$ & Reference \\
\hline $\begin{array}{l}\text { Homozygous, } \\
\text { Frameshift } \\
\text { (p.A214SfsX72) }\end{array}$ & + & 30 & $\mathrm{~F}$ & Negative & $\begin{array}{l}\text { Type I, } 3 \\
\text { years }\end{array}$ & $\begin{array}{l}\text { Bilateral, } 7 \\
\text { years }\end{array}$ & Bilateral, 20 years & $\begin{array}{l}\text { Abnormal MRI: } \\
\text { cerebellar ataxia } \\
\text { Dysuria and intermittent } \\
\text { urinary incontinence }\end{array}$ & Our study \\
\hline & & & & & & & & Gastro duodenal hypokinesia & \\
\hline $\begin{array}{l}\text { Homozygous, } \\
\text { Frameshift (F883fsX951) }\end{array}$ & + & 42 & $\mathrm{M}$ & Negative & $?, 8$ years & $?, 12$ years & ?, 10 years & Abnormal EEG & {$[21]$} \\
\hline $\begin{array}{l}\text { Heterozygous } \\
\text { Frameshift (F883fsX950) }\end{array}$ & + & 20 & $\mathrm{~F}$ & Negative & ?, 7 years & ?, 11 years & $?, 13$ years & Cataract & {$[21]$} \\
\hline $\begin{array}{l}\text { Homozygous, } \\
\text { nonsense (p.Q486X) }\end{array}$ & + & 35 & M & Negative & ?, 7 years & $\begin{array}{l}\text { Bilateral, } 10 \\
\text { years }\end{array}$ & $\begin{array}{l}\text { Ability deceased } \\
\text { in the late third } \\
\text { decade of life, } 30 \\
\text { years }\end{array}$ & $\begin{array}{l}\text { Hydronephrosis and } \\
\text { hydroureter } \\
\text { Hyperreflexia in the lower } \\
\text { limbs }\end{array}$ & {$[34]$} \\
\hline $\begin{array}{l}\text { Heterozygous, } \\
\text { nonsense (p.Q194X) }\end{array}$ & + & 31 & $\mathrm{~F}$ & Negative & $?, 5$ years & ?, 16 years & ?,? & $\begin{array}{l}\text { Retarded puberty } \\
\text { Cataract diabetic retinopathy }\end{array}$ & {$[18]$} \\
\hline $\begin{array}{l}\text { Compound heterozygous, } \\
\text { missense } \\
\text { (p.F350I + p.G674R) }\end{array}$ & - & 42 & M & Negative & $\begin{array}{l}\text { Type I, } 28 \\
\text { years }\end{array}$ & $\begin{array}{l}\text { Bilateral, } 42 \\
\text { years }\end{array}$ & Bilateral & No & {$[10]$} \\
\hline $\begin{array}{l}\text { Heterozygous, } \\
\text { missense (p.H313Y) }\end{array}$ & - & 24 & M & Negative & $\begin{array}{l}\text { Type I, } 10 \\
\text { years }\end{array}$ & $\begin{array}{l}\text { Bilateral, } 24 \\
\text { years }\end{array}$ & Bilateral, 2.5 years & Left-sides glaucoma, 24 years & {$[10]$} \\
\hline
\end{tabular}

DM: Diabetes Mellitus; OA: Optic Atrophy; HI: Hearing Impairment; MRI: Magnetic resonance imaging; EEG: Electroencephalogram; ?: Not indicated.

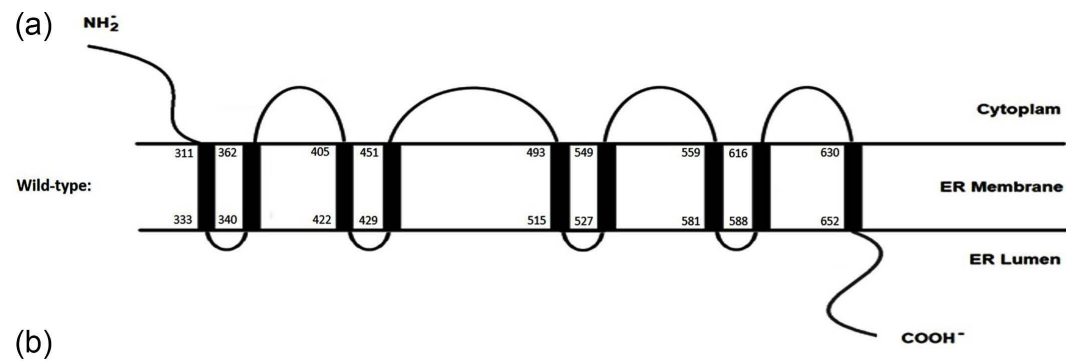

(b)

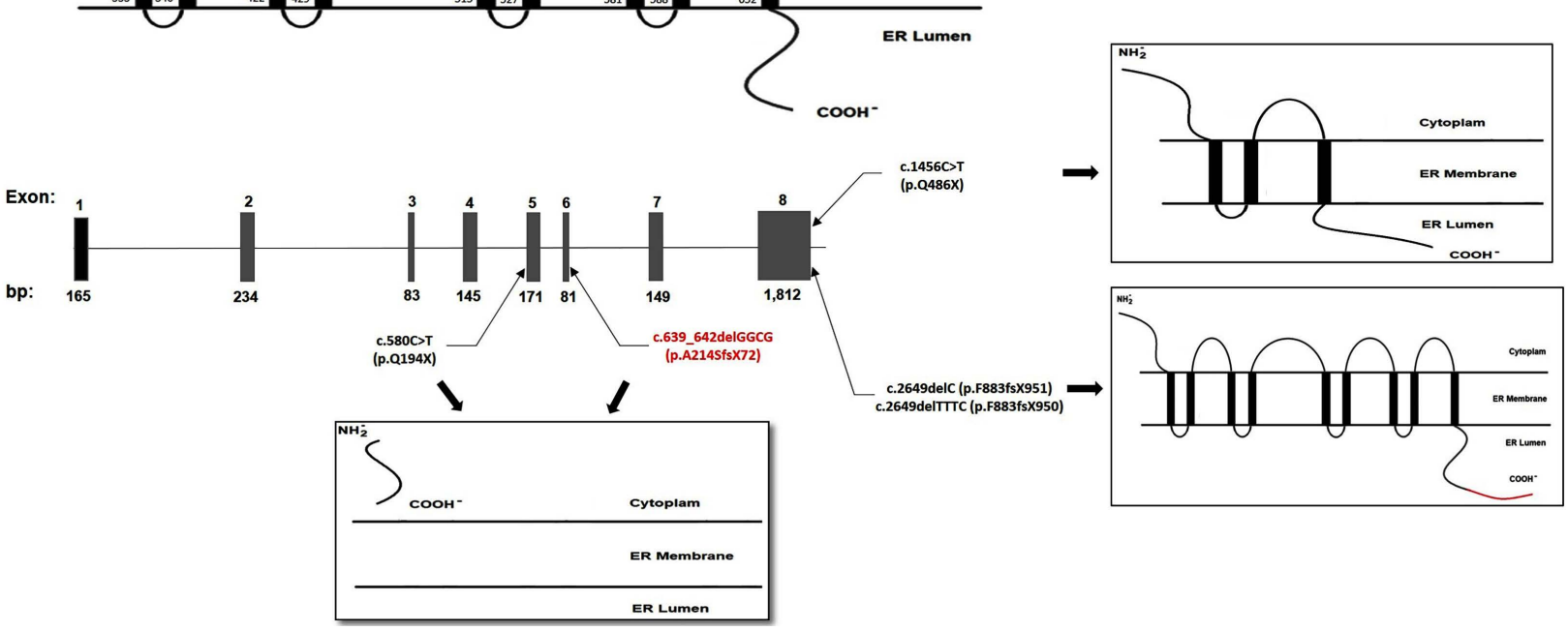

Figure 3. Schematic illustration of five mutated WFS1 proteins caused by truncating mutations in Wolfram syndrome (a) Full-length human WFS1 is 890 aa long and contains nine transmembrane domains. (b) Schematic representation of WFS1 derivatives caused by truncated mutations including the novel p.A214SfsX72 mutation. 


\section{3. p.A214SfsX72 Loss of Function Consequences}

It is hard to predict exactly the effect of the loss of function mutations on the biological and molecular functions of wolframin that are clearly elucidated. Indeed, it has been reported that wolframin protein is likely involved in calcium homeostasis and is a negative regulator of ER stress and described as a target for CaM. Wolframin has been shown to interact with $\mathrm{CaM}$ in a $\mathrm{Ca}^{2+}$ dependent manner and proteomic approaches lead to identifying $\mathrm{Ca}^{2+} / \mathrm{CaM}$-mediated signaling pathway [8]. Indeed, the N-terminal cytoplasmic domain (E90-W186) in wolframin has been identified to be the CaM-binding region and likely forms a compact conformation [8]. Based on the effect of the frameshift mutation p.A214SfsX72 on the deletion of all domains and part of the N-terminal tail, we aimed to study the effect on its interaction with CaM using molecular modeling. The 3-D protein modeling shows that the p.A214SfsX72 mutation caused misfolding of WFS1 protein and abolishing the CaM binding site and then impairing the WFS1-CaM binding and interaction. These findings strongly suggest not only the pathogenic effect of the novel mutation but also that CaM binding could be important in regulating wolframin function, and therefore, wolframin may participate in a novel $\mathrm{Ca}^{2+}$ signaling transduction system. In addition, CaM-AC8 complex plays a key role in integrating the cAMP-Ca ${ }^{2+}$ [23], and Kondo et al. showed that WFS1 is important for glucose and GLP1 (Glucagon-like peptide-1) induced cAMP (Cyclic adenosine monophosphate) production [24]. WFS1 mutations impairing these interactions lead to reduction of cAMP activity and insulin mRNA synthesis. Thus, mutated WFS1 translocates back to the ER, contributing to the UPR (Unfolded Protein Response) activation and to the inhibition of insulin biosynthesis and secretion [25] (Figure 4). This consequence on $\beta$-Cell function in Wolfram Syndrome was also analyzed by functional study performed on fibroblast cells from patients with different WFS1 mutations. Indeed, results revealed that WFS1 deficient $\beta$-Cells showed increased levels of ER stress molecules and decreased insulin content. In addition, when these WFS1 deficient $\beta$-Cells were exposed to experimental ER stress, their insulin processing was impaired and were unable to enhance insulin secretion in response to glucose and other secretagogues [15]. Besides, Plaas et al., described a $W f s 1$ mutant rats (Wfs1-ex5-KO232 rats) in which exon 5 of the $W f s 1$ gene is deleted resulting in a loss of 27 amino acids from the protein. This $W f s 1$ mutant rat model mimics the human WFS conditions and showed progressive glucose intolerance, leading to diabetes mellitus, glycosuria, and hyperglycemia, accompanied by decreased glucose-stimulated insulin secretion [14].

Restoring ER homeostasis required functional Wolframin which is a UPR component that is activated by the accumulation of misfolded or unfolded proteins [26]. Mutations in WFS1 generate wolframin deficiency, resulting in unfolded protein accumulation and inappropriate UPR activation which might lead to apoptosis [27]. Infancy-onset WFS had been proved to be caused by $\beta$-cell loss and Langerhans islets atrophy, via ER stress [28] (Figure 4). Besides, during ER 


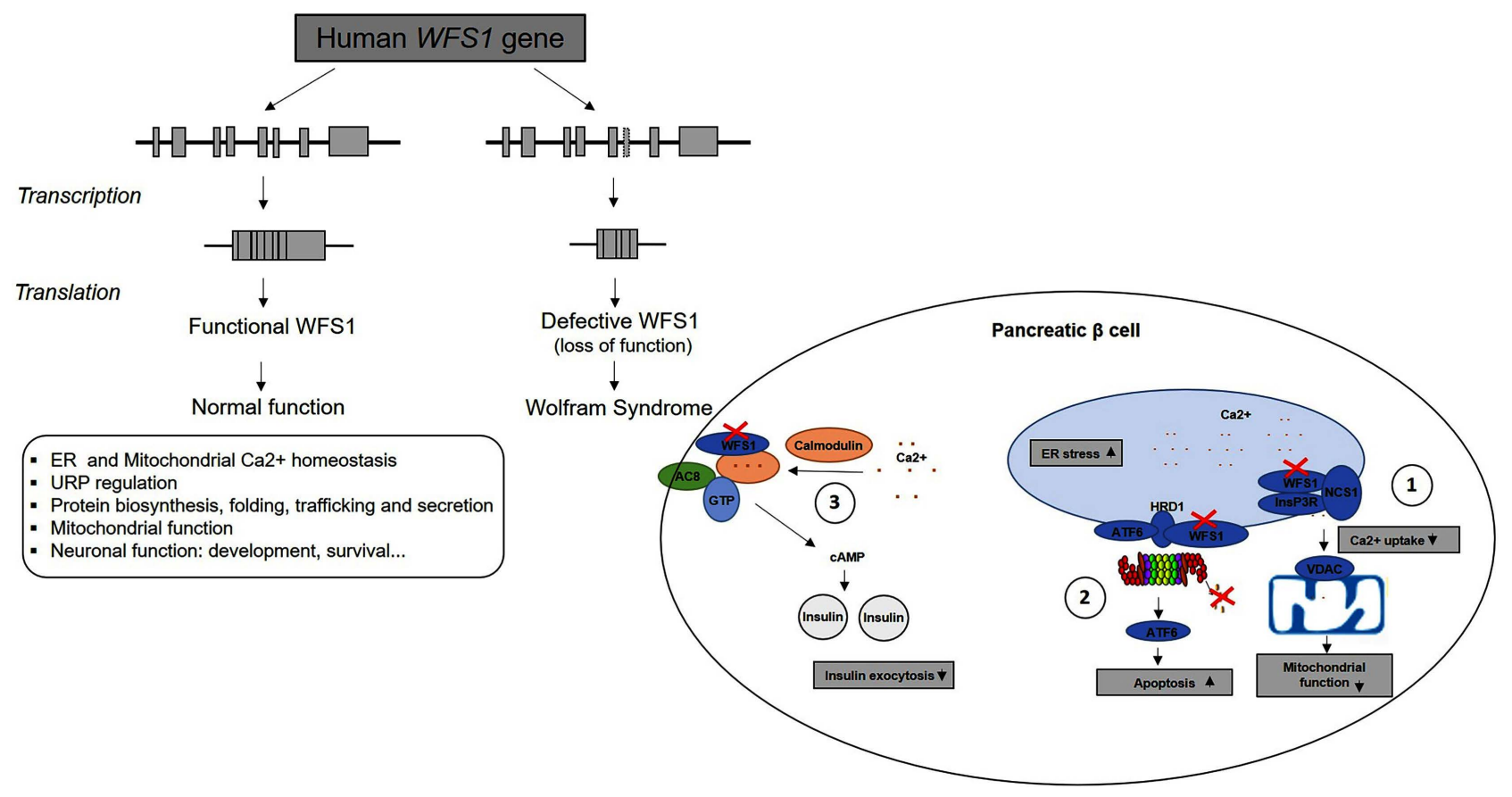

Figure 4. Schematic illustration of the structure and function of WFS1 under normal and disease conditions. In Wolfram syndrome, mutations in WFS1 gene causing loss of function of the WFS1 protein leads to 1) NCS1 degradation, no formation of the complex with IP3R1 and decreased ER-mitochondria $\mathrm{Ca}^{2+}$ transfer. Therefore, the WFS1/NCS1/IP3R1 complex and VDAC1 are non-functional, $\mathrm{Ca}^{2+}$ cannot be properly transferred from the ER to mitochondria and activate the TCA cycle leading to mitochondrial respiratory chain deficiency. 2) Mutated WFS1 is unable to recruit the ER transcription factor ATF6 $\alpha$ to the E3 ligase HRD1 under ER stress conditions. ATF6 $\alpha$ escapes from the proteasome-dependent degradation, leading to chronic hyperactivation of ATF6 $\alpha$ signaling and apoptosis. 3) Mutated WFS1 compromises complex formation between AC8 and calmodulin which alters cAMP activity, responsible for increasing insulin secretion and the protein folding demand. When the ER stress response is activated, WFS1 translocates back to the ER, contributing to the UPR. This concomitantly inhibits insulin secretion and biosynthesis.

stress, WFS1 also plays a crucial role in regulating ATF6 $\alpha$ (Activating Transcription Factor 6 alpha). Indeed, in WFS, ATF $6 \alpha$ is released from WFS1 and escapes from the proteosome-dependent degradation and it translocates from the ER membrane to the nucleus. ATF6 $\alpha$ upregulates stress signaling targets and its hyperactivation is involved in $\beta$-cell apoptosis [25] (Figure 4). A functional study using HEK295T cell line showed that knocking down WFS1 up-regulates the mRNA levels of GRP78, XBP1, and CHOP, which are ER stress-related molecules. Furthermore, TUNEL assay and flow cytometry analysis showed that the transfection with wild-type WFS1 expression plasmid decreased cell apoptosis compared to mutant WFS1 expression plasmid. WFS1 mutant activated the unfolded protein response (UPR) pathway and inducing dysregulated ER stress signaling and increasing the cell apoptosis [13]. These findings could explain the first and early manifestation in our proband with WFS. Also, WFS is characterized by clinical manifestations affecting several organs as in mitochondrial diseases has been also described with mitochondrial respiratory chain deficiency due to the role of WFS1 loss of function in the decrease of ER-mitochondria $\mathrm{Ca}^{2+}$ transferred and NCS1 (Neuronal Calcium Sensor 1) degradation [29]. In fact, 
mutated WFS1 is involved in mitochondrial dysfunction and $\mathrm{Ca}^{2+}$ uptake. In healthy cells, WFS1 binds to NCS1 (Neuronal Calcium Sensor 1) and form with IP3R1 (Inositol 1, 4,5-Triphosphate Receptor, type 1) a complex to activate ER-mitochondria $\mathrm{Ca}^{2+}$ transfer via VDAC1 (Voltage-Dependent Anion Channel 1). $\mathrm{Ca}^{2+}$ can be properly transferred from the ER to mitochondria and activate the TCA cycle and mitochondrial respiratory chain (Figure 4).

Under certain metabolic conditions, La Morgia et al. discovered calcium mishandling between the ER and the mitochondria, resulting in mitochondrial dysfunction. They revealed that wolframin protein is abundant in mitochondrial-associated ER membranes and that WFS1 protein is totally absent in patient-derived fibroblasts. They stated that a loss of function might result in improper calcium influx within mitochondria [30].

Given these findings, we suggested that the combination of impaired functions of WFS1 including improper WFS1-CaM interaction, $\mathrm{Ca}^{2+}$ uptake, mitochondrial dysfunction, and apoptosis under the ER stress could be involved in the severe phenotype and early onset of WFS in our patient.

\section{Conclusion}

In summary, we report severe WFS phenotype in a patient affected by novel WFS1 homozygous frameshift mutation resulting in total removal of the nine transmembrane domains which lead to a complete loss of WFS1 function. We also demonstrated by 3D modelling its effect on the CaM-WFS1 impaired interaction and we discuss its possible consequence in pancreatic $\beta$-cells dysfunction such as mitochondrial respiratory chain deficiency, apoptosis and insulin secretion/biosynthesis inhibition, and its role in the early onset of diabetes.

\section{Funding}

This research did not receive any specific grant from funding agencies in the public, commercial, or not-for-profit sectors.

\section{Acknowledgements}

The authors thank the patient and her family for their collaboration. We also thank the doctors of the Endocrinology department-Mahdia's hospital andCHU Hedi Chaker Sfax hospital.

\section{Conflicts of Interest}

The authors declare no conflicts of interest regarding the publication of this paper.

\section{References}

[1] Strom, T.M., et al. (1998) Diabetes Insipidus, Diabetes Mellitus, Optic Atrophy and Deafness (DIDMOAD) Caused by Mutations in a Novel Gene (Wolframin) Coding for a Predicted Transmembrane Protein. Human Molecular Genetics, 7, 2021-2028. 
https://doi.org/10.1093/hmg/7.13.2021

[2] Smith, C.J.A., Crock, P.A., King, B.R., Meldrum, C.J. and Scott, R.J. (2004) Phenotype-Genotype Correlations in a Series of Wolfram Syndrome Families. Diabetes Care, 27, 2003-2009. https://doi.org/10.2337/diacare.27.8.2003

[3] Cryns, K., et al. (2003) Mutational Spectrum of the WFS1 Gene in Wolfram Syndrome, Nonsyndromic Hearing Impairment, Diabetes Mellitus, and Psychiatric Disease. Human Mutation, 22, 275-287. https://doi.org/10.1002/humu.10258

[4] Fukuoka, H., Kanda, Y., Ohta, S. and Usami, S. (2007) Mutations in the WFS1 Gene Are a Frequent Cause of Autosomal Dominant Nonsyndromic Low-Frequency Hearing Loss in Japanese. Journal of Human Genetics, 52, Article No. 6. https://doi.org/10.1007/s10038-007-0144-3

[5] Hofmann, S., Philbrook, C., Gerbitz, K.-D. and Bauer, M.F. (2003) Wolfram Syndrome: Structural and Functional Analyses of Mutant and Wild-Type Wolframin, the WFS1 Gene Product. Human Molecular Genetics, 12, 2003-2012.

https://doi.org/10.1093/hmg/ddg214

[6] Li, L., Venkataraman, L., Chen, S. and Fu, H. (2020) Function of WFS1 and WFS2 in the Central Nervous System: Implications for Wolfram Syndrome and Alzheimer's Disease. Neuroscience \& Biobehavioral Reviews, 118, 775-783. https://doi.org/10.1016/j.neubiorev.2020.09.011

[7] Torkamandi, S., Rezaei, S., Mirfakhraie, R., Bayat, S., Piltan, S. and Gholami, M. (2020) A Homozygous Missense Mutation of WFS1 Gene Causes Wolfram's Syndrome without Hearing Loss in an Iranian Family (A Report of Clinical Heterogeneity). Journal of Clinical Laboratory Analysis, 34, e23358. https://doi.org/10.1002/jcla.23358

[8] Yurimoto, S., et al. (2009) Identification and Characterization of Wolframin, the Product of the Wolfram Syndrome Gene (WFS1), as a Novel Calmodulin-Binding Protein. Biochemistry, 48, 3946-3955. https://doi.org/10.1021/bi900260y

[9] Hatanaka, M., et al. (2011) Wolfram Syndrome 1 Gene (WFS1) Product Localizes to Secretory Granules and Determines Granule Acidification in Pancreatic $\beta$-Cells. Human Molecular Genetics, 20, 1274-1284. https://doi.org/10.1093/hmg/ddq568

[10] Zhang, Y., Feng, L., Kong, X., Wu, J., Chen, Y. and Tian, G. (2019) Novel Mutations and the Ophthalmologic Characters in Chinese Patients with Wolfram Syndrome. Orphanet Journal of Rare Diseases, 14, Article No. 190. https://doi.org/10.1186/s13023-019-1161-y

[11] Cano, A., et al. (2007) Identification of Novel Mutations in WFS1 and Genotype-Phenotype Correlation in Wolfram Syndrome. American Journal of Medical Genetics Part A, 143, 1605-1612. https://doi.org/10.1002/ajmg.a.31809

[12] Rohayem, J., et al. (2011) Diabetes and Neurodegeneration in Wolfram Syndrome: A Multicenter Study of Phenotype and Genotype. Diabetes Care, 34, 1503-1510. https://doi.org/10.2337/dc10-1937

[13] Gong, Y., Xiong, L., Li, X., Su, L. and Xiao, H. (2021) A Novel Mutation of WFS1 Gene Leading to Increase ER Stress and Cell Apoptosis Is Associated an Autosomal Dominant Form of Wolfram Syndrome Type 1. BMC Endocrine Disorders, 21, 76. https://doi.org/10.1186/s12902-021-00748-Z

[14] Plaas, M., et al. (2017) Wfs1-Deficient Rats Develop Primary Symptoms of Wolfram Syndrome: Insulin-Dependent Diabetes, Optic Nerve Atrophy and Medullary Degeneration. Scientific Reports, 7, Article No. 10220. https://doi.org/10.1038/s41598-017-09392-x 
[15] Shang, L., et al. (2014) $\beta$-Cell Dysfunction Due to Increased ER Stress in a Stem Cell Model of Wolfram Syndrome. Diabetes, 63, 923-933. https://doi.org/10.2337/db13-0717

[16] Tanji, Y., Yamaguchi, S., Ishigaki, Y., Katagiri, H., Oka, Y. and Ishihara, H. (2015) DPP-4 Inhibition Ameliorates Pancreatic $\beta$-Cell Failure and Improves Glucose Tolerance in the Mouse Model of Wolfram Syndrome. Journal of Diabetes Mellitus, 5, Article No. 2. https://doi.org/10.4236/jdm.2015.52009

[17] Lewin, H.A. and Stewart-Haynes, J.A. (1992) A Simple Method for DNA Extraction from Leukocytes for Use in PCR. BioTechniques, 13, 522-524.

[18] Kopanos, C., et al. (2019) VarSome: The Human Genomic Variant Search Engine. Bioinformatics, 35, 1978-1980. https://doi.org/10.1093/bioinformatics/bty897

[19] de Heredia, M.L., Clèries, R. and Nunes, V. (2013) Genotypic Classification of Patients with Wolfram Syndrome: Insights into the Natural History of the Disease and Correlation with Phenotype. Genetics in Medicine, 15, 497-506. https://doi.org/10.1038/gim.2012.180

[20] Grenier, J., et al. (2016) WFS1 in Optic Neuropathies: Mutation Findings in Nonsyndromic Optic Atrophy and Assessment of Clinical Severity. Ophthalmology, 123, 1989-1998. https://doi.org/10.1016/j.ophtha.2016.05.036

[21] Kobayashi, M., et al. (2018) WFS1 Mutation Screening in a Large Series of Japanese Hearing Loss Patients: Massively Parallel DNA Sequencing-Based Analysis. PLoS ONE, 13, e0193359. https://doi.org/10.1371/journal.pone.0193359

[22] Hansen, L., et al. (2005) Mutation Analysis of the WFS1 Gene in Seven Danish Wolfram Syndrome Families; Four New Mutations Identified. European Journal of Human Genetics, 13, Article No. 12. https://doi.org/10.1038/sj.ejhg.5201491

[23] Masada, N., Schaks, S., Jackson, S.E., Sinz, A. and Cooper, D.M.F. (2012) Distinct Mechanisms of Calmodulin Binding and Regulation of Adenylyl Cyclases 1 and 8. Biochemistry, 51, 7917-7929. https://doi.org/10.1021/bi300646y

[24] Kondo, M., et al. (2018) Activation of GLP-1 Receptor Signalling Alleviates Cellular Stresses and Improves Beta Cell Function in a Mouse Model of Wolfram Syndrome. Diabetologia, 61, 2189-2201. https://doi.org/10.1007/s00125-018-4679-y

[25] Fonseca, S.G., et al. (2010) Wolfram Syndrome 1 Gene Negatively Regulates ER Stress Signaling in Rodent and Human Cells. Journal of Clinical Investigation, 120, 744-755. https://doi.org/10.1172/JCI39678

[26] Hetz, C., Zhang, K. and Kaufman, R.J. (2020) Mechanisms, Regulation and Functions of the Unfolded Protein Response. Nature Reviews Molecular Cell Biology, 21, Article No. 8. https://doi.org/10.1038/s41580-020-0250-Z

[27] Oslowski, C.M. and Urano, F. (2010) A Switch from Life to Death in ER Stressed $\beta$ Cells. Diabetes, Obesity and Metabolism, 12, 58-65. https://doi.org/10.1111/j.1463-1326.2010.01277.x

[28] Fonseca, S.G., Burcin, M., Gromada, J. and Urano, F. (2009) Endoplasmic Reticulum Stress in Beta Cells and Development of Diabetes. Current Opinion in Pharmacology, 9, 763-770. https://doi.org/10.1016/j.coph.2009.07.003

[29] Angebault, C., et al. (2018) ER-Mitochondria Cross-Talk Is Regulated by the $\mathrm{Ca}^{2+}$ Sensor NCS1 and Is Impaired in Wolfram Syndrome. Science Signaling, 11, eaaq1380. https://doi.org/10.1126/scisignal.aaq1380

[30] La Morgia, C., et al. (2020) Calcium Mishandling in Absence of Primary Mitochondrial Dysfunction Drives Cellular Pathology in Wolfram Syndrome. Scientific Reports, 10, Article No. 4785. https://doi.org/10.1038/s41598-020-67203-2 
[31] Colosimo, A., et al. (2003) Molecular Detection of Novel WFS1 Mutations in Patients with Wolfram Syndrome by a DHPLC-Based Assay. Human Mutation, 21, 622-629. https://doi.org/10.1002/humu.10215

[32] Inoue, H., et al. (1998) A Gene Encoding a Transmembrane Protein Is Mutated in Patients with Diabetes Mellitus and Optic Atrophy (Wolfram Syndrome). Nature Genetics, 20, 143-148. https://doi.org/10.1038/2441

[33] Hong, J., et al. (2009) The Novel Compound Heterozygous Mutations, V434del and W666X, in WFS1 Gene Causing the Wolfram Syndrome in a Chinese Family. Endocrine, 35, 151-157. https://doi.org/10.1007/s12020-009-9145-7

[34] Haghighi, A., Haghighi, A., Setoodeh, A., Saleh-Gohari, N., Astuti, D. and Barrett, T.G. (2013) Identification of Homozygous WFS1 Mutations (p.Asp211Asn, p.Gln486*) Causing Severe Wolfram Syndrome and First Report of Male Fertility. European Journal of Human Genetics, 21, 347-351.

https://doi.org/10.1038/ejhg.2012.154 